\title{
Nanoscale Reduction of Graphene Oxide under Ambient Conditions
}

\author{
Austin C. Faucett and Jeffrey M. Mativetsky* \\ Department of Physics, Applied Physics and Astronomy, Binghamton University, \\ Binghamton, New York, 13902, USA.
}

\begin{abstract}
:
Localized voltage-induced reduction, initiated by a conductive atomic force microscope probe under ambient conditions, is used to pattern electrically conductive reduced graphene oxide (rGO) regions in electrically insulating graphene oxide (GO). This method is shown to reduce single and multiple layer GO on ultra-flat Au substrates with feature sizes as small as $4.0 \mathrm{~nm}$, with the reduction resolution depending strongly on humidity and number of GO layers. In situ current levels during reduction are used to track reaction kinetics, which follow a rate-limited process where the generation and transport of hydrogen ions are the primary rate limiting steps. Tip-enhanced Raman spectroscopy is used to map the nanoscale structure and local disorder in voltage-reduced GO and rGO single sheets. It is found that reduction of GO causes a decrease in tip-enhanced Raman scattering intensity in both the D-band and Gband, but the $\mathrm{D} / \mathrm{G}$ intensity ratio remains unchanged following reduction, indicating that defects are not introduced by the reduction process.
\end{abstract}

* Corresponding author. E-mail: jmativet@binghamton.edu (Jeffrey M. Mativetsky) 


\section{Introduction}

Graphene oxide (GO) and reduced graphene oxide (rGO) are emerging as materials with extensive electronic and optoelectronic applications [1,2]. GO has a graphenelike carbon lattice decorated with oxygen-containing groups, primarily hydroxyl and epoxy groups along the basal plane, and carbonyl and carboxyl groups populating edge sites [3]. These groups disrupt the $\mathrm{sp}^{2}$-conjugated carbon network, rendering GO electrically insulating [4]. Reduction - the removal of oxygen containing groups via thermal, chemical, or electrochemical processes - has been shown to recover electrical conductivity [5]. Reduction can also tune the chemical and optical properties of GO, making GO a versatile material for chemical or biological sensing, organic electronic devices, and energy storage applications [1,3,5-13].

A further attractive feature of GO is that it can be readily dispersed in an aqueous medium and processed from solution. Spin, drop, or spray coating of GO onto substrates, and subsequent reduction into rGO, provides a simple means of fabricating flexible and transparent electrodes $[3,14,15]$. Common approaches used for reduction, however, involve high temperatures that are incompatible with flexible substrates, or use harsh reducing agents that are not practical due to environmental concerns $[4,5]$. Alternate methods of reduction, such as electrochemical, photolytic, or less hazardous chemical approaches, are being actively pursued [13,16,17]. Beyond large area reduction of GO, there is also significant interest in patterning two-dimensional materials, such as GO and graphene, to produce circuitry with precisely engineered conductive and insulating regions [9-11,18-20].

Voltage-induced reduction under ambient conditions has emerged as a promising approach that enables selective localized reduction while avoiding the above issues 
that are associated with conventional reduction methods [21-23]. Voltage-induced GO reduction was first demonstrated by applying a voltage between a conductive atomic force microscope (C-AFM) probe and an electrode on the sample surface [21,22]. This process depends on a water meniscus that forms around the probe under ambient conditions and acts as a localized electrochemical cell [18,21,23]. Upon application of a voltage between the probe and counter-electrode, water molecules are split, generating $\mathrm{H}^{+}$ions that react with GO's functional groups, leading to reduction $[13,23]$. Voltage-induced reduction can be used for patterning conductive rGO regions in insulating GO and has potential to be scaled up by use of patterned electrode arrays $[22,23]$. Nevertheless, this reduction method is relatively unexplored beyond these initial studies, and much is still unknown about the reduction mechanisms, reaction kinetics, and resolution limits.

In this work, we examine ambient voltage-induced reduction of GO in the as of yet unexplored vertical geometry, whereby a GO sheet or film is sandwiched between a conductive substrate and a conductive AFM probe (Figure 1a) [19]. We demonstrate that in this geometry, rGO can be controllably patterned with feature sizes as small as $4.0 \mathrm{~nm}$. This resolution is significantly higher than previously shown in the lateral geometry $(\sim 1 \mu \mathrm{m})$ [21], or using other scanning probe methods that involve elevated temperatures or hydrogen gas exposure $(12-80 \mathrm{~nm})$ [24,25]. To gain insight into the mechanisms and kinetics of ambient voltage-induced GO reduction, we monitor in situ current levels during reduction and put forward a kinetic model. We also link local conductivity, gleaned from C-AFM, to structural information identified by highresolution tip-enhanced Raman spectroscopy (TERS). 


\section{Experimental Section}

\subsection{Sample Preparation}

Commercially obtained graphene oxide (Harp engineering) was dispersed in deionized water at concentrations of $1 \mathrm{mg} / \mathrm{ml}$ and $2 \mathrm{mg} / \mathrm{ml}$. These dispersions were stirred for periods $>30$ days to fully exfoliate the GO. Solutions were spin-coated onto ultra-flat template-stripped gold substrates, fabricated following procedures outlined in the literature [26]. The $1 \mathrm{mg} / \mathrm{ml} \mathrm{GO}$ solution was spin-coated at $1500 \mathrm{RPM}$ to obtain isolated GO sheets; the $2 \mathrm{mg} / \mathrm{ml} \mathrm{GO}$ solution was spin-coated at 1500RPM for fewlayer films and at 800 RPM to obtain thicker multi-layer GO films. Substrates were pre-treated with oxygen plasma for 3 minutes to promote adhesion. Verification of single or double layer GO, and thickness of few layer films were measured by tapping mode AFM.

\subsection{C-AFM Characterization and Reduction}

Characterization of GO films by AFM and C-AFM was implemented with a Combiscope 1000 AFM system from AIST-NT. Pt-coated silicon cantilevers (Budget Sensors ContE-g; nominal $\mathrm{k}=0.2 \mathrm{~N} / \mathrm{m}, \mathrm{f}_{0}=13 \mathrm{kHz}$, probe radius $<25 \mathrm{~nm}$ ) were used for C-AFM current mapping, current-voltage measurements, and tip-induced reduction. Spring constants were determined for each tip using the Sader Method [27]. An applied force of $10 \mathrm{nN}$ and a sample bias of $50 \mathrm{mV}$ were used for C-AFM current imaging. During point-by-point imaging, the probe is lifted out of contact before being moved to the next measurement point, where the tip reengages in contact with the sample at the set force. For all C-AFM measurements, a $10 \mathrm{M} \Omega$ resistor was added in series with the sample in order to limit current levels and preserve tip integrity. All 
characterization was carried out under ambient conditions with humidity monitored using a digital humidity/temperature sensor.

The standard procedure for voltage-induced reduction of isolated points was: landing the probe with a contact force of $10 \mathrm{nN}$ while applying a $-4.0 \mathrm{~V}$ bias to the sample, with the tip grounded. Patterning of rGO lines was done with either close $(<10$ nm point spacing) point-by-point reductions or by scanning the probe at $<1 \mu \mathrm{m} / \mathrm{s}$ while applying a constant voltage. Rectangular regions of rGO were patterned by raster scanning the probe at a rate of $1 \mu \mathrm{m} / \mathrm{s}$ while holding a constant $-4.0 \mathrm{~V}$ bias.

\subsection{TERS Characterization}

TERS was done using an OmegaScope 1000 from AIST-NT. Etched gold cantilevers, sourced from AIST-NT, were used for TERS mapping in a topillumination setup with a $200 \mu \mathrm{W}, 637 \mathrm{~nm}$ incident laser [28,29]. TERS maps consisted of spectra collected over a grid with a $50 \mathrm{~nm}$ spacing. Raman spectra were collected with 200 ms acquisition times while the tip was in contact with the sample with controlled force of $15 \mathrm{nN}$. After acquisition, the tip was lifted and then moved to the next point where the process was repeated. Linear background subtraction of spectra was done in the vicinity of the $\mathrm{D}$ and $\mathrm{G}$ peaks.

\section{Results and Discussion}

This study focuses on characterizing voltage-induced reduction in a vertical electrode geometry. A diagram of the experimental setup is shown in Figure 1a. The Pt-coated AFM probe contacts the GO with an applied force of $10 \mathrm{nN}$. During contact, a several volt negative bias is applied to the Au substrate to reduce the GO, while 
monitoring current through the GO (or rGO, upon reduction). This process is repeated at multiple specified locations to produce arrays of reduced spots. Then, a noninvasive low bias $(50 \mathrm{mV})$ is applied between tip and substrate to image the same area with C-AFM and track changes in local conductivity caused by reduction. These changes can be correlated with surface features as seen in AFM topography or lateral force data recorded simultaneously to the C-AFM current maps. To produce isolated spots and to ensure that each reduction event is independent from one another, the spacing between reduced spots is kept significantly larger than spot diameter. This approach allows the collection of large data sets with significant statistical robustness. To minimize effects of substrate roughness, ultra-flat template-stripped gold (TS-Au) [26] was used as the conductive substrate with an RMS surface roughness of $0.30 \mathrm{~nm}$ measured over a $1 \mu \mathrm{m}$ x $1 \mu \mathrm{m}$ area.
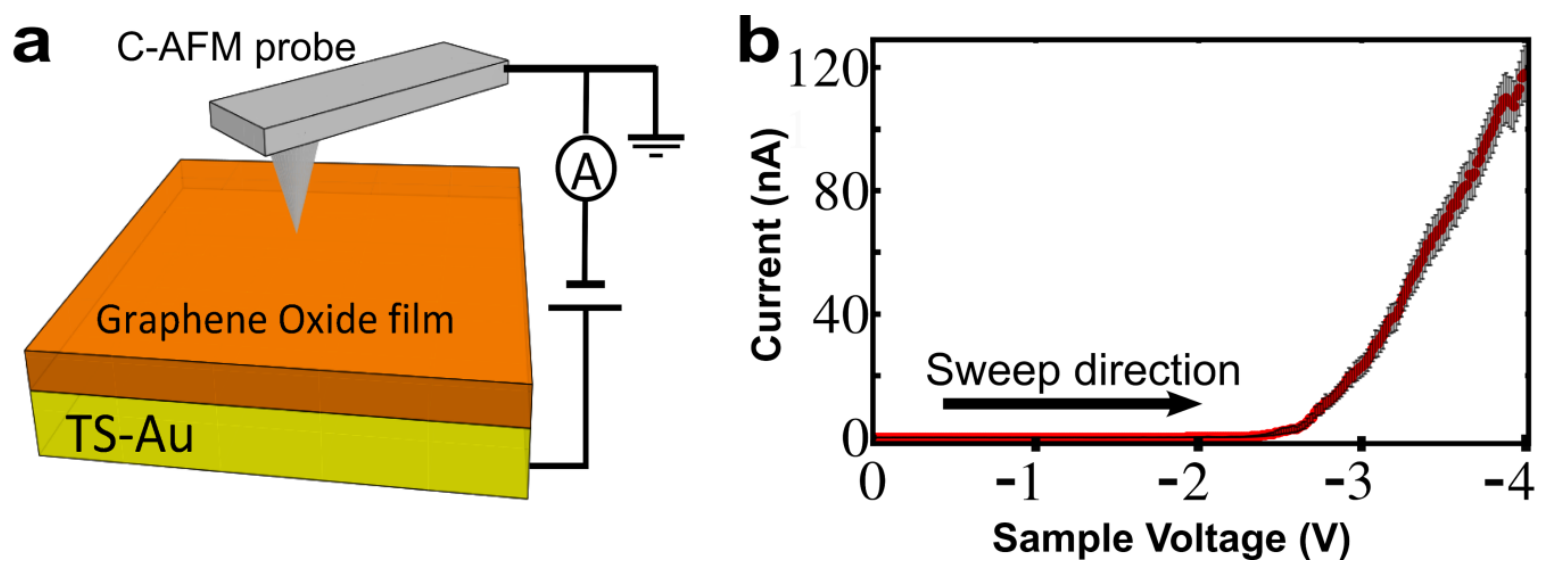

Figure 1. a) Setup employed for voltage-induced reduction. b) Current, averaged over twenty voltage sweeps at different sample locations, used to extract a reduction onset voltage of $-2.5 \pm 0.2 \mathrm{~V}$. 
When sweeping the sample voltage to a several volt negative bias, the current rapidly increases at the onset of reduction, as shown in Figure 1b. This increase in current is due to the drastic decrease in electrical resistance upon reduction of GO to rGO. The average of 20 current-voltage sweeps, from 0 to $-4 \mathrm{~V}$ over 5 seconds, was used to find a $-2.5 \pm 0.2 \mathrm{~V}$ threshold voltage for single layer GO reduction. Few-layer GO's reduction onset voltage was also found to be in this range. This reduction threshold is lower than the value previously reported for lateral reduction $(-3.6 \pm 0.3$ V), potentially due to the significantly larger inter-electrode spacing when performing lateral measurements $[21,23]$. It was also found that the time dependence of reduction (to be discussed in later sections) must be taken into account to accurately determine onset voltage. Accurate onset determination requires slow sweeps ( $>1$ second for single layer or $>10$ seconds for few layer films), otherwise kinetics will affect the results.

Reduction experiments were performed on single layer GO sheets $(0.9 \pm 0.1 \mathrm{~nm}$ thick), GO bilayers (1.9 \pm 0.2nm thick), continuous few-layer GO films (4-6 nm thick) and many layer GO films (12-15 nm thick). A C-AFM current map of a region with bare gold, single layer GO, and bilayer GO is shown in Figure 2a. Current mapping of single layer unreduced GO reveals isolated sub-10nm diameter conductive domains, supporting findings of heterogeneity in GO, characterized by 3-6 nm sized $\mathrm{sp}^{2}$ conjugated domains surrounded by predominantly oxygen-decorated $\mathrm{sp}^{3}$ carbon regions [4,30,31]. Bilayer GO shows a suppression of current from the conductive domains to within detectible limits ( $<100 \mathrm{fA}$ ), probably due to a mismatch of $\mathrm{sp}^{2}$ cluster locations in the layers, rendering the surface insulating throughout. Current 
maps of unmodified few layer regions also show no measureable currents with a sample bias of $50 \mathrm{mV}$.

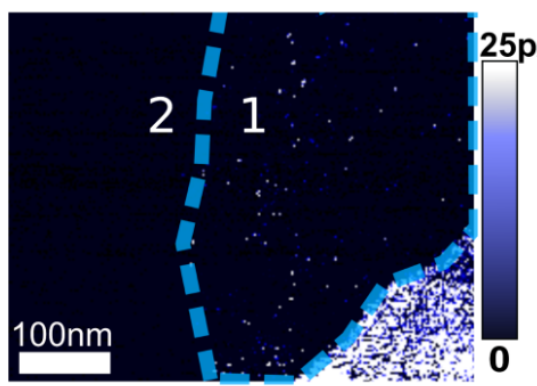

a

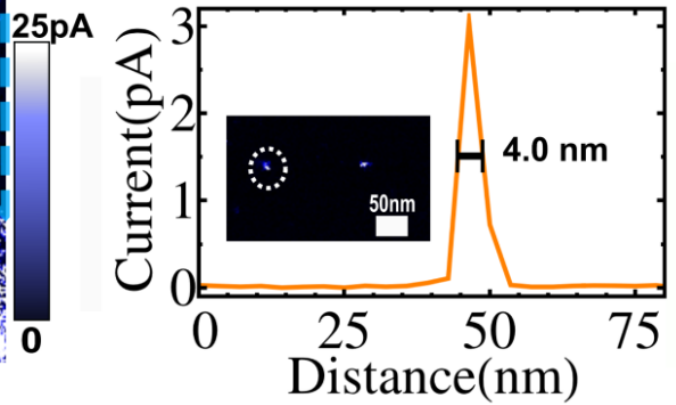

b

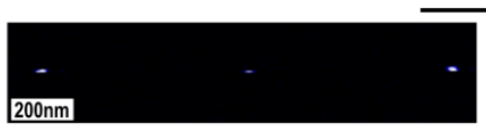

C Increasing Humidity

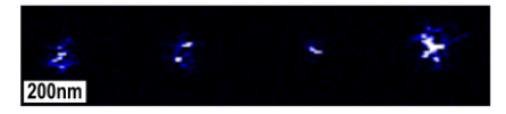

d

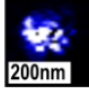

a

e

Figure 2. a) C-AFM current map of single layer GO, bilayer GO, and bare Au regions. The outlined single layer region (1) exhibits sub-10nm conductive domains; in the bilayer region (2), the film is uniformly electrically insulating. b) A cross section of an rGO spot (outlined in the inset) with a FWHM of $4.0 \mathrm{~nm}$. The inset shows a CAFM current map of two reduced spots, measured using the point-by-point C-AFM imaging mode. c-e) Current maps showing the effect of increasing humidity from c) $<20 \% \mathrm{RH}$ to d) $40 \% \mathrm{RH}$ to e) $70 \% \mathrm{RH}$ on few-layer rGO spot size.

Application of a negative voltage of magnitude greater than the threshold $(-2.5 \pm 0.2 \mathrm{~V})$ resulted in local reduction at the probe location. Prior work performed in a lateral geometry was limited to micrometer-sized features [21]. Here, using a vertical geometry, we observe localized reduction with nanoscale feature size. Figure $2 \mathrm{~b}$ shows the current profile of an rGO spot patterned in a GO bilayer during low humidity conditions $(<20 \% \mathrm{RH})$, demonstrating a full-width at half maximum 
(FWHM) of $4.0 \mathrm{~nm}$. This is presently the highest resolution demonstrated for rGO patterning. As this feature size is near the resolution limit of the C-AFM technique, the rGO spot size may in fact be smaller than measured. The tip contact diameter, estimated to be $3.0 \mathrm{~nm}$ (see Supporting information), sets the upper limit on imaging resolution. The data shown in Figure $2 \mathrm{~b}$ was recorded using point-by-point C-AFM in which the probe is retracted between pixels, thereby reducing lateral forces. Using standard C-AFM imaging, a FWHM feature width of $6 \mathrm{~nm}$ was determined (see Supporting Information).

The shape and size of the reduced spots is observed to be dependent on the ambient humidity, number of GO layers, and the conditions of the local film or substrate. As shown in Figure 2(c-e), increasing ambient humidity causes reduced spots to increase in size dramatically. For few layer GO films, rGO spots reduced at low humidity $(<20 \% \mathrm{RH})$ have a FWHM diameter of $19 \pm 6 \mathrm{~nm}$ while spots reduced at high humidity ( $\sim 80 \% \mathrm{RH})$ increase in size to $120 \pm 80 \mathrm{~nm}$ FWHM. This observed trend can be explained by the relative amount of water in the vicinity of the AFM probe at different humidity levels. As previously mentioned, and to be discussed in further detail later, during voltage-induced reduction, $\mathrm{H}^{+}$ions generated by water splitting react with GO's oxygen-bearing functional groups. Low relative humidity decreases the size of the water meniscus around the AFM tip apex, effectively decreasing the area subject to $\mathrm{H}^{+}$ions generated in the meniscus [18,32]. Reduction at low to moderate (20 - 40\%) humidity was observed to produce the most consistently sized rGO spots, with variability in size increasing with humidity.

Film thickness also has a significant effect on spot size, causing average FWHM of rGO spots (reduced in $<20 \%$ humidity) to increase from $8 \pm 2 \mathrm{~nm}$ in bilayer films to 
$19 \pm 6 \mathrm{~nm}$ in few layer films to $40 \pm 10 \mathrm{~nm}$ in many layer films. We hypothesize that a thinner film (i.e. closer tip-substrate separation) curtails lateral spread of the electric field between the tip and substrate, causing the transport of $\mathrm{H}^{+}$ions involved in reduction to proceed with limited lateral spreading.

Local GO and substrate features also influence rGO spot size and uniformity. As seen in the topmost reduced spot in Figure 3(a-b), reduction propagates along sheet wrinkles, suggesting enhanced electrochemical activity in these structures compared to flat GO. Sensitivity to substrate morphology is observed when GO is reduced near a substrate defect, such as the scratch-like feature indicated by an arrow in Figure 3a and Figure 3b. The reduced spot at the defect is larger than nearby reduced spots and follows the general shape of the underlying defect. Another observed feature in this process is a change in height of $0.2-0.5 \mathrm{~nm}$ upon reduction of single layer GO, consistent with other reported reduction methods [24,25]. Furthermore, lateral force measurements, providing a friction map of the scanned area, show lower friction for rGO than GO (see Supporting Information), in accordance with studies of rGO reduced by other means [24,33]. 

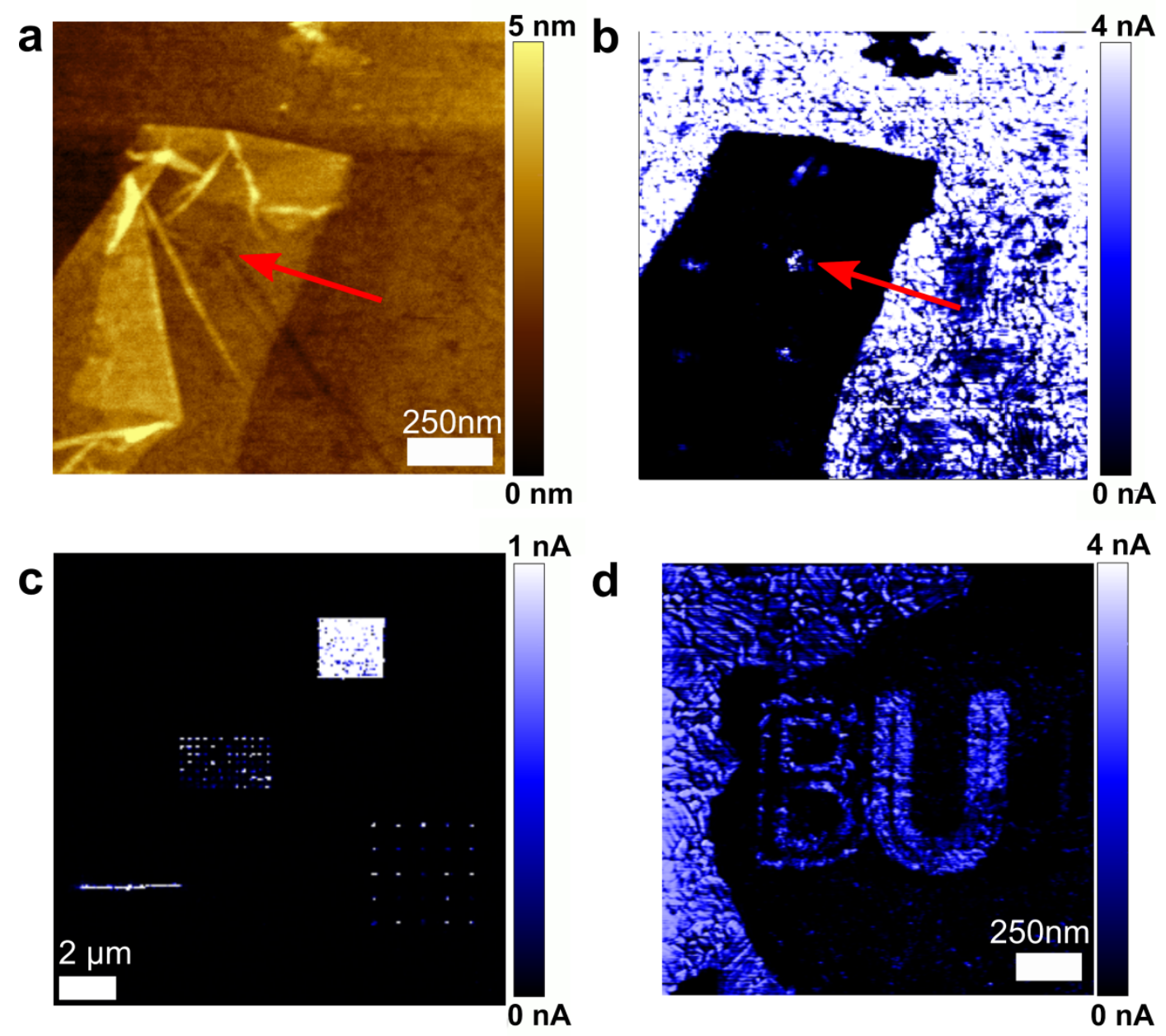

Figure 3. a) AFM topography of a GO sheet on Au with a substrate defect (indicated by the arrow). b) Current map showing voltage-reduced spots on the same GO sheet. c) Current map of arrays of rGO spots, a line, and a $2 \mu \mathrm{m}$ x $2 \mu \mathrm{m}$ rGO region in few layer GO. d) Current map of an rGO pattern in a single layer GO sheet.

Conductive regions of arbitrary design, such as arrays of reduced spots, continuous lines, and extended areas, can be patterned in GO, as shown in Figure 3c and 3d. There is practical interest in patterning narrow lines of $\mathrm{rGO}$, as sub-10 $\mathrm{nm}$ graphene nanoribbons have been shown to behave as semiconductors due to quantum 
confinement and edge effects opening a transport gap [34,35]. Voltage-induced reduction allows patterning of rGO lines that approach this limit. At moderate humidity (40\% RH), a line width of $70 \pm 20 \mathrm{~nm}$ is obtained when using a point-bypoint patterning method. At low humidity $(<20 \% \mathrm{RH})$, line resolution increases drastically, giving rGO lines with 12-30 nm FWHM width (see Supporting Information).
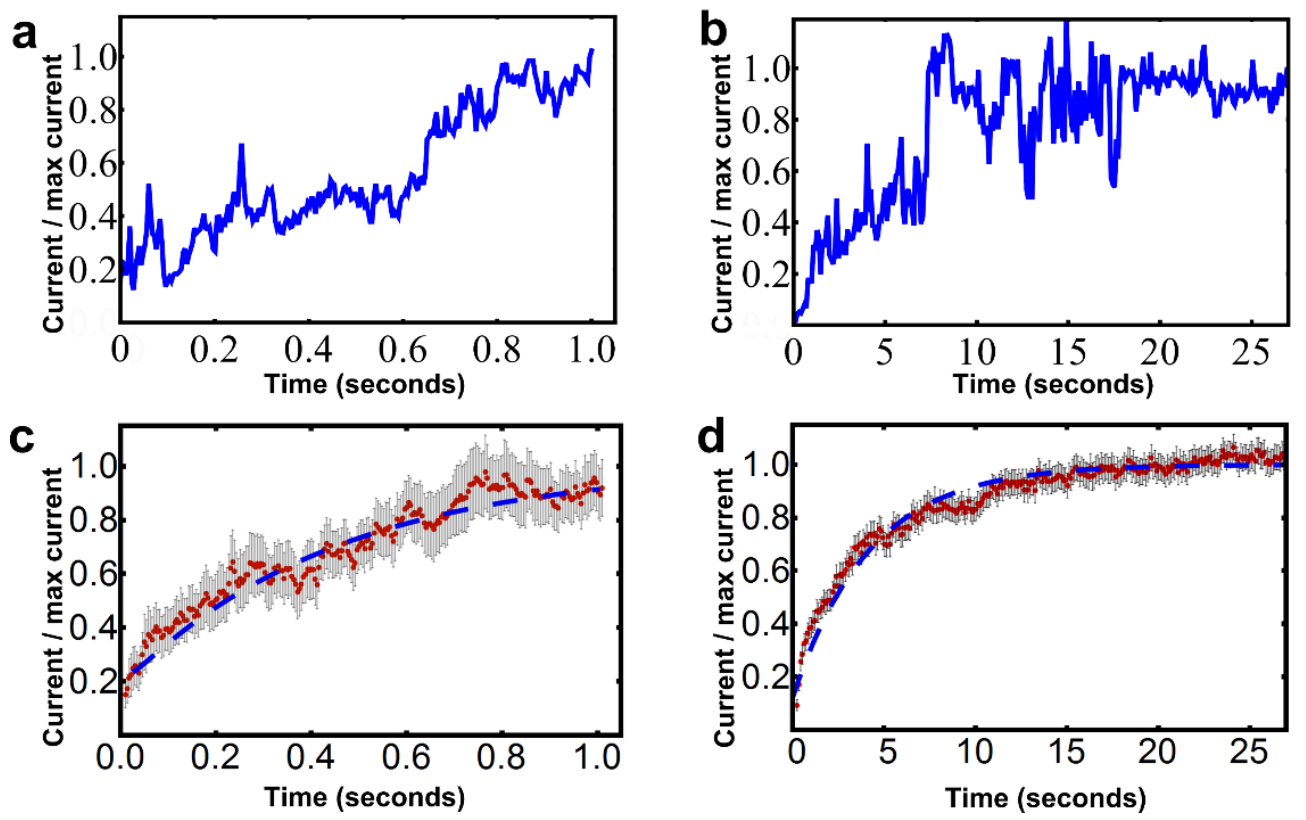

Figure 4. Transient normalized current measured during reduction of a) single layer GO, and b) few-layer GO. Average transient normalized current for c) twenty different reduction spots in single layer GO and d) one-hundred spots in few-layer GO. Dashed lines show fits to a kinetic model. 
To investigate the mechanisms of voltage-induced reduction, populations of isolated reduction events were analysed. Transient current levels collected during reduction provide insight into reduction kinetics [36]. For a constant voltage, the current level will scale inversely with the resistance, allowing the current to indicate the level of GO reduction. We observe that individual in situ current versus time curves, shown in Figure $4 \mathrm{a}$ and $4 \mathrm{~b}$, respectively, for single layer and few-layer reduction, are highly variable, exhibiting discreet jumps and plateaus before saturating. The stochastic nature of individual curves is likely due to the heterogeneous distribution of functionalized groups and defects in GO $[1,16]$. When averaged over multiple data sets recorded at different sample locations, the time dependence of current shows smooth rate-limited behavior (Figure 4c and 4d). Single layer GO undergoes reduction quickly, having current levels reaching 95\% of their maximum after 1 second, as shown in Figure 4c. Few layer GO exhibits saturated current levels after 10 seconds (Figure 4d), indicating that film thickness is highly important in the kinetics of reduction.

We propose a kinetic model to describe the time-dependence of reduction, based on Alberty-Miller (AM) integrated rate equations [37,38]. Our model assumes that the kinetic process begins with an initial state [A], proceeds reversibly to an intermediate state [B], and then proceeds irreversibly to a final state [C] (see Supporting Information). Other possible reaction schemes were tested, but did not produce the same quality fit to the data as the one presented. As will be discussed later, besides producing the best fit to the data, the chosen kinetic framework corresponds well with available electrochemical pathways. The time-dependent degree of reduction is represented as the quantity of final product [C], with the condition that it has a 
minimum occurring at time $\mathrm{t}=0$, for unreduced GO, and has a maximum at a later time, for complete reduction. We employ the current measured during application of a constant voltage as the experimental measure of the degree of reduction.

The averaged in situ current versus time curves were fit with the AM equations. Rate constants for single GO sheets, few layer GO films, and many layer GO films are shown in Table 1 for data taken in a moderate humidity (40 $\pm 5 \% \mathrm{RH})$ environment. We first note that the rate $\mathrm{k}_{\mathrm{AB}}$, undergoes a slight increase with film thickness. The rate for the reverse process, $k_{\mathrm{BA}}$, is 2.5 times higher for few layer films in comparison with single layer GO, and larger yet for many-layer films. The third process, with rate constant $\mathrm{k}_{\mathrm{BC}}$, decreases steeply, by two orders of magnitude, as GO film thickness increases from one layer to more than 10 layers, indicating that it is the primary rate limiting step.

Table 1. Kinetic parameters of reduction for different thickness GO films, reduced under $40 \pm 5 \%$ relative humidity.

\begin{tabular}{lccc}
\hline Sample & $\mathrm{k}_{\mathrm{AB}}$ & $\mathrm{k}_{\mathrm{BA}}$ & $\mathrm{k}_{\mathrm{BC}}$ \\
\hline Single Layer $(0.9 \pm 0.1 \mathrm{~nm})$ & $9.7 \pm 0.5$ & $34 \pm 3$ & $13 \pm 1$ \\
Few-Layer $(4-6 \mathrm{~nm})$ & $10.6 \pm 0.3$ & $85 \pm 9$ & $2.2 \pm 0.3$ \\
Many-Layer $(12-15 \mathrm{~nm})$ & $11.0 \pm 0.5$ & $110 \pm 10$ & $0.15 \pm 0.03$ \\
\hline
\end{tabular}


Associating these rate steps with electrochemical processes is the next step to identify the mechanisms of voltage-induced reduction. The reactions leading to reduction take place in the vicinity of the water meniscus at the probe-sample interface, and have been proposed to proceed according to reactions 1 and 2:

$$
\begin{aligned}
& \text { (1) } 2 \mathrm{H}_{2} \mathrm{O} \rightarrow 4 \mathrm{H}^{+}+\mathrm{O}_{2}+4 \mathrm{e}^{-} \\
& \text {(2) } \mathrm{aH}^{+}+\mathrm{be}^{-}+\mathrm{GO} \rightarrow \mathrm{rGO}+\mathrm{cH}_{2} \mathrm{O}
\end{aligned}
$$

We associate the first step of the reduction process, $A \rightarrow B$, with the generation of reactive ions within the water meniscus, described by reaction 1 . Subsequent reaction with functional groups, represented in our model by the process $\mathrm{B} \rightarrow \mathrm{C}$ and reaction 2 , leads to the final product, rGO. This reaction scheme is illustrated in Figure 5. Following reaction 1 that generates $\mathrm{H}^{+}$ions, the electric field between the AFM probe and Au substrate acts to transport $\mathrm{H}^{+}$ions towards the negatively biased Au electrode, to react with GO according to reaction 2 [21]. Reversion of the ions back to water or evolution of gaseous hydrogen is associated with the process $\mathrm{B} \rightarrow \mathrm{A}$, accounting for the fact that only some ions will react with GO groups. 


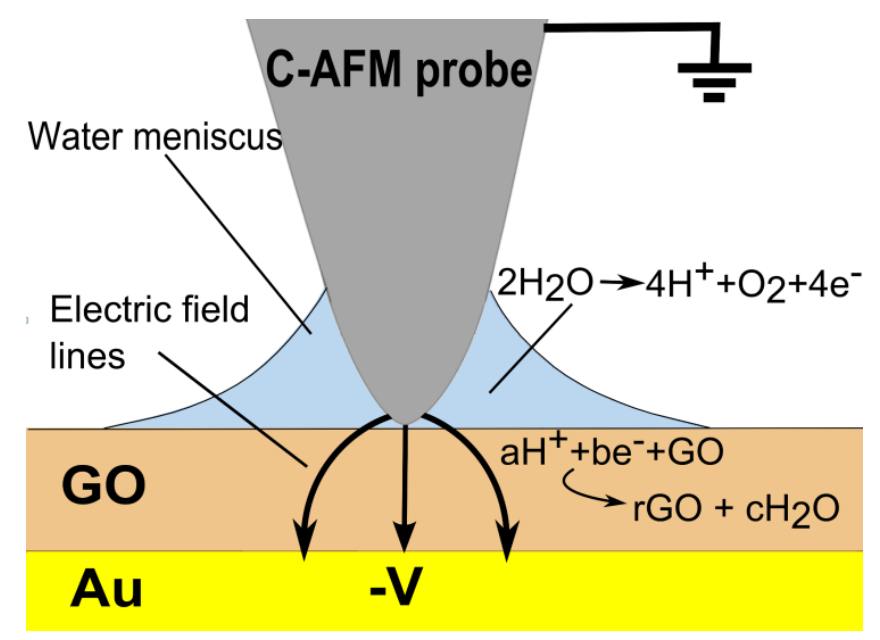

Figure 5. Schematic of the voltage induced reduction process: the tip-substrate voltage splits water to generate hydrogen ions in the water meniscus, and transports the ions to react with the oxygen containing groups that populate GO, resulting in local reduction.

The rate constant $\mathrm{k}_{\mathrm{AB}}$, associated with the generation of reactive ions, is similar for all three examined GO thicknesses, but increases slightly with thickness. This can be understood on the basis that both experiments were done at the same relative humidity (40 $\pm 5 \% \mathrm{RH})$ and voltage bias, resulting in a similar generation rate of ions. The increase in $\mathrm{k}_{\mathrm{AB}}$ with thickness is likely the result of a higher amount of intercalated water in the thicker films. Preliminary experiments indicate that an increase in humidity leads to an increase in $\mathrm{k}_{\mathrm{AB}}$, as the size of the water meniscus and hence the amount of water undergoing electrolysis increases [18]. The loss of $\mathrm{H}^{+}$ions is accounted for in the kinetic model by considering $\mathrm{A} \rightarrow \mathrm{B}$ as a reversible process. $\mathrm{k}_{\mathrm{BA}}$ effectively limits the reduction of GO via a mechanism proposed to be the combination of ions into gaseous hydrogen or back into water, processes that are more likely to occur as ions must travel further through the film. This description is 
consistent with the observed increase in $\mathrm{k}_{\mathrm{BA}}$ as film thickness is increased. The final step $\mathrm{B} \rightarrow \mathrm{C}$, with rate constant $\mathrm{k}_{\mathrm{BC}}$, is associated with the transport of $\mathrm{H}^{+}$ions to reaction sites in GO. This rate is strongly dependent on GO thickness because transport of $\mathrm{H}^{+}$is further impeded as GO thickness increases. This is because, first of all, as GO thickness increases, the electric field strength between the probe and Au decreases (due to the larger inter-electrode spacing), thus slowing $\mathrm{H}^{+}$transport. Secondly, the rate $\mathrm{k}_{\mathrm{BC}}$ will be slowed in thicker GO films because reactive $\mathrm{H}^{+}$ions must percolate across the GO layers to reach and react with deeper layers.

To further characterize the structure of voltage-reduced GO, we use TERS. TERS takes advantage of increased electric field intensity near a metal tip apex to both enhance Raman scattering from the area in the immediate vicinity of the tip, and enable sub-diffraction limited spatial resolution [39]. AFM topography and C-AFM current maps, shown in Figure 6a and 6b, respectively, show a patterned $2 \mu \mathrm{m} \times 2 \mu \mathrm{m}$ conductive rGO square in single-layer GO. Average TERS spectra (Figure 6c) in the GO and rGO areas demonstrate pronounced peaks at about $1350 \mathrm{~cm}^{-1}$ and $1600 \mathrm{~cm}^{-1}$ representing the $\mathrm{D}$ and the $\mathrm{G}$ bands, respectively $[4,17,21]$. The $\mathrm{G}$ band is due to scattering from $E_{2 g}$ phonon modes, characteristic of $\mathrm{sp}^{2}$ carbon networks, and the D band is associated with disorder caused by structural defects [3]. 
a
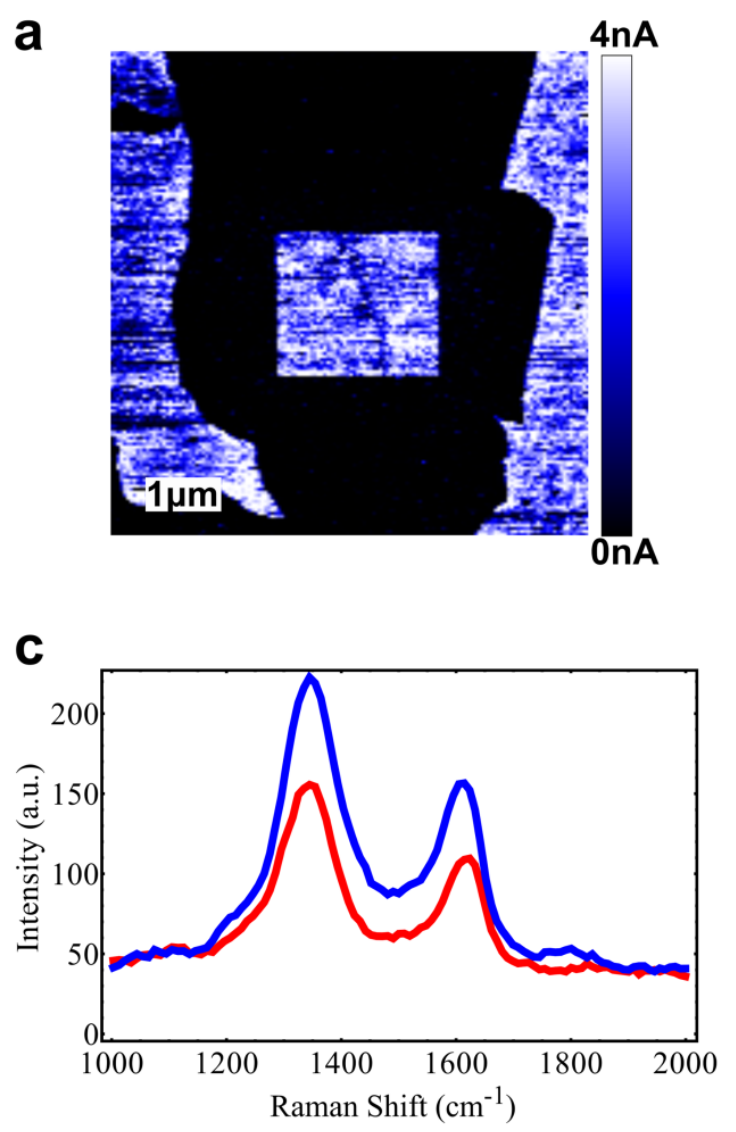

b

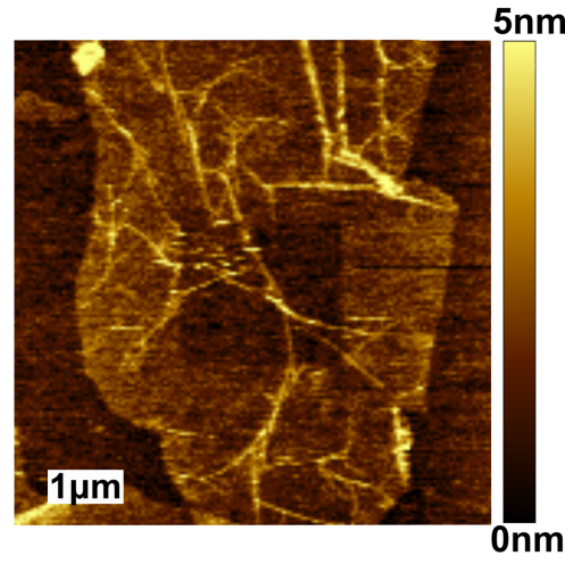

d

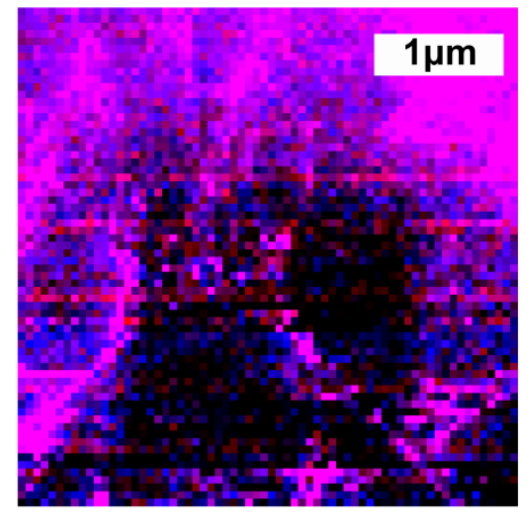

Figure 6. a) C-AFM current map and b) AFM topography of single layer GO with a 2 $\mu \mathrm{m}$ x $2 \mu \mathrm{m}$ reduced area. c) Average TERS spectrum (averaged over 900 spectra) for GO (blue) and rGO (red) regions. d) G-band TERS map of the center region in a) and b).

A higher intensity of the $\mathrm{D}$ band $\left(\mathrm{I}_{\mathrm{D}}\right)$ compared to the $\mathrm{G}$ band $\left(\mathrm{I}_{\mathrm{G}}\right)$ is observed in both reduced and unreduced GO spectra and is consistent with the observation of more prominent D bands than obtained with standard Raman for TERS data from graphene systems $[28,40,41]$. A 30\% decrease in $I_{D}$ and $I_{G}$, shown in Figure 6c, is observed in reduced regions. This stronger Raman signal in GO compared to rGO has been previously observed using a related technique, surface enhanced Raman spectroscopy (SERS) [42], however, without the spatial resolution offered by TERS [43]. The $\mathrm{I}_{\mathrm{G}}$ 
map, given in (figure 6d), shows contrast between single layer rGO and GO, owing to the reduced TERS signal in rGO. In the TERS map, local variations in intensity across both rGO and GO are observed. This result is consistent with other studies, where spatially heterogeneous oxygen functionalization is observed with high-resolution transmission electron microscopy and photoluminescence spectroscopy $[1,16,21]$. Wrinkled structures are visible in rGO and GO TERS maps, with significant contrast with respect to neighboring flat regions. The increased TERS signal at wrinkles is likely a result of the increased amount of $\mathrm{sp}^{2}$ regions and defects in the vicinity of the tip compared to flat regions. The ratio of the TERS-obtained D and G intensities $\left(\mathrm{I}_{\mathrm{D}} / \mathrm{I}_{\mathrm{G}}\right)$ is unchanged for voltage-reduced and unreduced GO. This result indicates that local defect density is similar for each region and that the $\mathrm{sp}^{2}$ domains in rGO are relatively undamaged by the voltage-induced reduction [40].

\section{Conclusion}

Localized voltage-induced reduction, initiated by a conductive atomic force microscope probe under ambient conditions, is demonstrated as a method for patterning conductive rGO regions in insulating GO with features sizes as small as $4.0 \mathrm{~nm}$. This minimum feature size is in the range used for opening a band gap in graphene [35,44], and could potentially be employed to modulate the electronic structure of rGO through similar quantum confinement mechanisms [1,45].

In situ current levels during reduction were used to study the kinetics of reduction with Alberty-Miller rate theory, providing insight into the rate-limiting processes during reduction. The transport of reactive hydrogen ions to functional groups in GO was determined to be a primary rate limiting step, particularly in thicker GO films. 
High-resolution TERS mapping was performed on patterned GO/rGO single sheet samples to characterize the dependence of Raman signals on local sheet structure. TERS mapping provided contrast between GO and rGO regions, and between flat and wrinkled regions, demonstrating the promise of TERS as a sensitive platform for local structural mapping of $\mathrm{GO}$ and related materials. The $\mathrm{I}_{\mathrm{D}} / \mathrm{I}_{\mathrm{G}}$ ratio following reduction was unchanged, indicating that voltage-induced reduction avoids introducing substantial defects.

\section{Acknowledgements}

We thank Andrey Krayev and AIST-NT for assistance with TERS and use of their facilities. We acknowledge the NSF (CMMI-1537648, CMMI-1429176) for financial support.

\section{References}

[1] Loh KP, Bao Q, Eda G, Chhowalla M. Graphene Oxide as a Chemically Tunable Platform for Optical Applications. Nat Chem 2010;2:1015-24. doi:10.1038/nchem.907.

[2] Brownson D, Kampouris D, Banks C. Graphene Electrochemistry: Fundamental Concepts Through to Prominent Applications. Chem Soc Rev 2012;41:6944. doi:10.1039/c2cs35105f.

[3] Chen D, Feng H, Li J. Graphene oxide: Preparation, functionalization, and electrochemical applications. Chem Rev 2012;112:6027-53. doi:10.1021/cr300115g. 
[4] Mattevi C, Eda G, Agnoli S, Miller S, Mkhoyan KA, Celik O, et al. Evolution of Electrical, Chemical, and Structural Properties of Transparent and Conducting Chemically Derived Graphene Thin Films. Adv Funct Mater 2009;19:2577. doi:10.1002/adfm.200900166.

[5] Dreyer DR, Park S, Bielawski CW, Ruoff RS. The Chemistry of Graphene Oxide. Chem Soc Rev 2010;39:228. doi:10.1039/b917103g.

[6] Jung I, Dikin DA, Piner RD, Ruoff RS. Tunable Electrical Conductivity of Individual Graphene Oxide Sheets Reduced at “Low” Temperatures. Nano Lett 2008;8:4283. doi:10.1021/nl8019938.

[7] Yang D, Velamakanni A, Bozoklu G, Park S, Stoller M, Piner RD, et al. Chemical Analysis of Graphene Oxide Films After Heat and Chemical Treatments by X-ray Photoelectron and Micro-Raman Spectroscopy. Carbon N Y 2009;47:145. doi:10.1016/j.carbon.2008.09.045.

[8] Zhu Y, Murali S, Cai W, Li X, Suk JW, Potts JR, et al. Graphene and Graphene Oxide: Synthesis, Properties, and Applications. Adv Mater 2010;22:3906. doi:10.1002/adma.201001068.

[9] Hong JY, Jang J. Micropatterning of Graphene Sheets: Recent Advances in Techniques and Applications. J Mater Chem 2012;22:8179. doi:10.1039/c2jm00102k.

[10] Bi YG, Feng J, Li YF, Zhang YL, Liu YS, Chen L, et al. Arbitrary Shape Designable Microscale Organic Light-Emitting Devices by Using Femtosecond Laser Reduced 
Graphene Oxide as a Patterned Electrode. ACS Photonics 2014;1:690.

doi:10.1021/ph500106f.

[11] He Q, Sudibya HG, Yin Z, Wu S, Li H, Boey F, et al. Centimeter-Long and LargeScale Micropatterns of Reduced Graphene Oxide Films: Fabrication and Sensing Applications. ACS Nano 2010;4:3201-8. doi:10.1021/nn100780v.

[12] Dreyer DR, Todd AD, Bielawski CW. Harnessing the Chemistry of Graphene Oxide. Chem Soc Rev 2014;43:5288. doi:10.1039/c4cs00060a.

[13] Kuila T, Mishra AK, Khanra P, Kim NH, Lee JH. Recent Advances in the Efficient Reduction of Graphene Oxide and its Application as Energy Storage Electrode Materials. Nanoscale 2013;5:52. doi:10.1039/c2nr32703a.

[14] Becerril HA, Mao J, Liu Z, Stoltenberg RM, Bao Z, Chen Y. Evaluation of SolutionProcessed Reduced Graphene Oxide Films as Transparent Conductors. ACS Nano 2008;2:463-70. doi:10.1021/nn700375n.

[15] Eda G, Nathan A, Wöbkenberg P, Colleaux F, Ghaffarzadeh K, Anthopoulos TD, et al. Graphene Oxide Gate Dielectric for Graphene-Based Monolithic Field Effect Transistors. Appl Phys Lett 2013;102:133108/1. doi:10.1063/1.4799970.

[16] McDonald MP, Eltom A, Vietmeyer F, Thapa J, Morozov Y V., Sokolov DA, et al. Direct Observation of Spatially Heterogeneous Single-Layer Graphene Oxide Reduction Kinetics. Nano Lett 2013;13:5777. doi:10.1021/nl402057j.

[17] Ambrosi A, Bonanni A, Sofer Z, Cross JS, Pumera M. Electrochemistry at Chemically Modified Graphenes. Chem Eur J 2011;17:10763. doi:10.1002/chem.201101117. 
[18] Kurra N, Reifenberger RG, Kulkarni GU. Nanocarbon-Scanning Probe Microscopy Synergy: Fundamental Aspects to Nanoscale Devices. ACS Appl Mater Interfaces 2014;6:6147. doi:10.1021/am500122g.

[19] Mativetsky JM, Loo YL, Samorì P. Elucidating the Nanoscale Origins of Organic Electronic Function by Conductive Atomic Force Microscopy. J Mater Chem C 2014;2:3118. doi:10.1039/c3tc32050b.

[20] Garcia R, Knoll AW, Riedo E. Advanced Scanning Probe Lithography. Nat Nanotechnol 2014;9:577. doi:10.1038/nnano.2014.157.

[21] Mativetsky JM, Treossi E, Orgiu E, Melucci M, Veronese GP, Samorì P, et al. Local Current Mapping and Patterning of Reduced Graphene Oxide. J Am Chem Soc 2010;132:14130. doi:10.1021/ja104567f.

[22] Ekiz OO, Urel M, Güner H, Mizrak AK, Dâna A. Reversible Electrical Reduction and Oxidation of Graphene Oxide. ACS Nano 2011;5:2475. doi:10.1021/nn1014215.

[23] Mativetsky JM, Liscio A, Treossi E, Orgiu E, Zanelli A, Samorì P, et al. Graphene Transistors Via In Situ Voltage-Induced Reduction of Graphene-Oxide Under Ambient Conditions. J Am Chem Soc 2011;133:14320. doi:10.1021/ja202371h.

[24] Wei Z, Wang D, Kim S, Kim SY, Hu Y, Yakes MK, et al. Nanoscale Tunable Reduction of Graphene Oxide for Graphene Electronics. Science (80- ) 2010;328:1373. doi:10.1126/science.1188119. 
[25] Zhang K, Fu Q, Pan N, Yu X, Liu J, Luo Y, et al. Direct Writing of Electronic Devices on Graphene Oxide by Catalytic Scanning Probe Lithography. Nat Commun 2012;3:1194. doi:10.1038/ncomms2200.

[26] Vogel N, Zieleniecki J, Köper I. As Flat as It Gets: Ultrasmooth Surfaces from Template-Stripping Procedures. Nanoscale 2012;4:3820. doi:10.1039/c2nr30434a.

[27] Sader JE, Chon JWM, Mulvaney P. Calibration of Rectangular Atomic Force Microscope Cantilevers. Rev Sci Instrum 1999;70:3967. doi:10.1063/1.1150021.

[28] Snitka V, Rodrigues RD, Lendraitis V. Novel Gold Cantilever for Nano-Raman Spectroscopy of Graphene. Microelectron Eng 2011;88:2759. doi:10.1016/j.mee.2011.02.046.

[29] Stadler J, Schmid T, Zenobi R. Nanoscale Chemical Imaging Using Top-Illumination Tip-Enhanced Raman Spectroscopy. Nano Lett 2010;10:4514. doi:10.1021/nl102423m.

[30] Azevedo J, Bourdillon C, Derycke V, Campidelli S, Lefrou C, Cornut R. Contactless Surface Conductivity Mapping of Graphene Oxide Thin Films Deposited on Glass with Scanning Electrochemical Microscopy. Anal Chem 2013;85:1812. doi:10.1021/ac303173d.

[31] Liu Z, Zhao X, Zhang X, Yan X, Wu Y, Chen Y, et al. Ultrafast Dynamics and Nonlinear Optical Responses from sp 2 - and sp 3 -Hybridized Domains in Graphene Oxide. J Phys Chem Lett 2011;2:1972. doi:10.1021/jz2008374. 
[32] Cambel V, Šoltýs J. The Influence of Sample Conductivity on Local Anodic Oxidation by the Tip of Atomic Force Microscope. J Appl Phys 2007;102:074315/1-074315/7. doi:10.1063/1.2794374.

[33] Mungse HP, Khatri OP. Chemically Functionalized Reduced Graphene Oxide as a Novel Material for Reduction of Friction and Wear. J Phys Chem C 2014;118:14394. doi:10.1021/jp5033614.

[34] Cai J, Ruffieux P, Jaafar R, Bieri M, Braun T, Blankenburg S, et al. Atomically Precise Bottom-Up Fabrication of Graphene Nanoribbons. Nature 2010;466:470. doi:10.1038/nature09211.

[35] Li X, Wang X, Zhang L, Lee S, Dai H. Chemically Derived, Ultrasmooth Graphene Nanoribbon Semiconductors. Science (80- ) 2008;319:1229. doi:10.1126/science.1150878.

[36] Kim TY, Ricci D, Zitti E Di, Cincotti S. A Study of the Transient Current During the Formation of Titanium Oxide Nanodots by AFM Anodic Oxidation. Surf Sci 2007;601:4910. doi:10.1016/j.susc.2007.08.017.

[37] Alberty RA, Miller WG. Integrated Rate Equations for Isotopic Exchange in Simple Reversible Reactions. J Chem Phys 1957;26:1231. doi:10.1063/1.1743498.

[38] Dagata JA, Perez-Murano F, Abadal G, Morimoto K, Inoue T, Itoh J, et al. Predictive Model for Scanned Probe Oxidation Kinetics. Appl Phys Lett 2000;76:2710. doi:10.1063/1.126451. 
[39] Stadler J, Schmid T, Zenobi R. Developments in and Practical Guidelines for TipEnhanced Raman Spectroscopy. Nanoscale 2012;4:1856. doi:10.1039/c1nr11143d.

[40] Ghislandi M, Hoffmann GG, Tkalya E, Xue L, With G De. Tip-Enhanced Raman Spectroscopy and Mapping of Graphene Sheets. Appl Spectrosc Rev 2012;47:371. doi:10.1080/05704928.2012.666773.

[41] Stadler J, Schmid T, Zenobi R. Nanoscale Chemical Imaging of Single-Layer Graphene. ACS Nano 2011;5:8442. doi:10.1021/nn2035523.

[42] Yu X, Cai H, Zhang W, Li X, Pan N, Luo Y, et al. Tuning Chemical Enhancement of SERS by Controlling the Chemical Reduction of Graphene Oxide Nanosheets. ACS Nano 2011;5:952-8. doi:10.1021/nn102291j.

[43] Yang H, Hu H, Ni Z, Poh CK, Cong C, Lin J, et al. Comparison of Surface-Enhanced Raman Scattering on Graphene oxide, Reduced Graphene Oxide and Graphene Surfaces. Carbon N Y 2013;62:422. doi:10.1016/j.carbon.2013.06.027.

[44] Han M, Özyilmaz B, Zhang Y, Kim P. Energy Band-Gap Engineering of Graphene Nanoribbons. Phys Rev Lett 2007;98:206805/1. doi:10.1103/PhysRevLett.98.206805.

[45] Zhou X, Lu G, Qi X, Wu S, Li H, Boey F, et al. A Method for Fabrication of Graphene Oxide Nanoribbons from Graphene Oxide Wrinkles. J Phys Chem C 2009;113:19119. doi:10.1021/jp9079298. 